\title{
Aerodynamic performance of a flyable flapping wing rotor with passive pitching angle variation
}

\author{
Si Chen ${ }^{1}$, Le Wang ${ }^{1 *}$, Yuanyuan $\mathrm{He}^{2^{*}}$, Mingbo Tong ${ }^{3}$, Yingjun $\mathrm{Pan}^{5}$, Bing $\mathrm{Ji}^{4}$, Shijun Guo ${ }^{5}$
}

\begin{abstract}
The present work was based on an experimental study on the aerodynamic performance of a flapping wing rotor (FWR) and enhancement by passive pitching angle variation (PPAV) associated with powered flapping motion. The PPAV (in this study $10^{\circ} \sim 50^{\circ}$ ) was realized by a specially designed sleeve-pin unit as part of a U-shape flapping mechanism. Through experiment and analysis, it was found that the average lift produced by an FWR of PPAV was $>100 \%$ higher than the baseline model, the same FWR of a constant pitching angle $30^{\circ}$ under the same input power. It was also noted that the lift-voltage relationship for the FWR of PPAV was almost linear and the aerodynamic efficiency was also over $100 \%$ higher than the baseline FWR when the input voltage was under 6V. The aerodynamic lift or efficiency of the FWR of PPAV can be also increased significantly by reducing the weight of the wings. An FWR model was fabricated and achieved vertical take-off and free flight powered by $9 \mathrm{~V}$ input voltage. The mechanism of PPAV function provides a feasible solution for aerodynamic improvement of a bio-inspired FWR and potential application to micro-air-vehicles (MAVs).
\end{abstract}

Index Terms- Flapping wing rotor (FWR), passive pitching angle variation (PPAV), aerodynamic performance, MAVs.

\section{INTRODUCTION}

$\mathrm{F}$ lying animals usually show particularly great manoeuvrability with its precise control of the flapping wing [1], [2]. Therefore, inspired by the insects or birds, the design of bio-inspired flapping wing (FW) and its aerodynamic study has attracted great attention over the past several decades [3]-[5]. Stanley et al [6] designed a $13 \mathrm{~g}$ ornithopter and realize its autonomous flight control with a 1.0 gram control electronics integrated with a microcontroller. The ornithopter can fly toward a target and land within a radius of $0.5 \mathrm{~m}$ without remote assistance. Lau et al [7] presented the design, analysis

\footnotetext{
Manuscript received Month $x x, 2 x x x$; revised Month $x x, \quad x x x x$; accepted Month $x, x x x x$. This work is supported by the following funding organizations in China: The National Natural Science Foundation of China (Grant No.11972079); The National Key Research and Development Program of China (Grant No. 2018YFB1305400). (Corresponding author: Le Wangle, Yuanyuan He)

Si Chen and Le Wang are with College of Mechanical and Electrical Engineering, WenZhou University, Wenzhou 325035, China (e-mail: 20200316@wzu.edu.cn; 20200315@wzu.edu.cn).
}

and characterization of a compliant thoracic mechanism that saves inertial power for flapping-wing micro air vehicles. With the capability of elastic energy storage, in comparison with the rigid-body flapping mechanism, their compliant thoracic mechanism saves power expenditure ranging from $20 \%$ up to $30 \%$ under the same thrust production.

Apart from the above experimental researches on the flapping wing, there are also many theoretical findings on the effect of passive deformation on the flapping wing's lift through numerical simulation. Stowers and Lentink [8] measured the unfolding kinematics of a flapping wing mechanism with passive wing morphing and constructed a numerical model of the unfolding process based on rigid body dynamics, contact models and aerodynamic correlations. They found that the morphing wings are possibly more energy efficient and lightweight. Zheng et al [9] used high-speed videogrammetry to capture the wing kinematics and the deformation of a Painted Lady butterfly (Vanessa cardui) in untethered forward flight and obtained its thrust and lift by using a high-fidelity threedimensional unsteady Navier-Stokes flow solver. The simulations show that the twist-only-wing model recovers much of the performance of the observed butterfly wing, demonstrating that wing-twist, and not camber is key to forward flight in these insects. Arabagi et al [10] develops a numerical simulation tool for designing the passive rotation flapping wing mechanisms. Although there exists tiny discrepancies compared with the experimental results, their simulation is able to predict the kinematics and the lift curves of the flapping wing. Yan et al [11] optimized the wing-kinematics for a hovering micro-air-vehicle (MAV) by projecting the problem down to a finite dimensional space of design variables. They found that the conventional models for pitching wings are not adequate as they predict considerably high rotational lift and too little power

Yuanyuan $\mathrm{He}$ is within the School of Aerospace Engineering, Beijing Institute of Technology, Beijing 100081, China (e-mail: appleyuanyuan@bit.edu.cn)

Mingbo Tong is with College of Aerospace Engineering, Nanjing University of Aeronautics and Astronautics, Nanjing 210016, China (email: tongw@nuaa.edu.cn).

Bing Ji is with School of Control Science and Engineering, ShanDong University, Jinan 250002, China (e-mail: $\underline{b . j i @ s d u . e d u . c n)}$

Shijun Guo and Yingjun Pan are within the School of Aerospace, Transport and Manufacturing, Cranfield University, Cranfield MK43 OAL, UK (e-mail: s.guo@cranfield.ac.uk; y.pan@cranfield.ac.uk). 
requirements, which makes the optimizer, unrealistically, leans toward almost pure rotational motion with little flapping. In addition, quasi-steady modelling overestimates the generated lift and, which leads to a more optimal, but unrealistic, performance. Therefore efficient unsteady modelling is essential in design optimization of flapping-wing micro-airvehicles.

Based on the traditional insect-like or bird-like flapping wings, a novel configuration with combined rotary and flapping motion called flapping wing rotor (FWR) was proposed [12]. The rotary motion is generated by the phenomenon of inverse Karmen vortex. In nature, the fishes are found to swipe their tails to move forward by utilizing the phenomenon of inverse Karmen vortex. It was found that this novel FWR of optimal kinematics of motion can produce significantly higher aerodynamic efficiency and lift coefficient than insect-like flapping wings in low Reynolds number and can achieve ideal Strouhal number around 0.3 along the whole wing [13], [14]. Although the lift efficiency of the FWR is as large as that of the rotorcraft, the larger lift coefficients is found for the FWR.

In previous study, research results have shown that passive twist of a flapping wing or a rotorcraft should be beneficial to the lift and efficiency [15]-[17]. Ghommem et al [18] investigated the role of morphing on flight dynamics of two birds by simulating the flow over rigid and morphing wings that have the characteristics of two different birds (the Giant Petrel and Dove Prion). They found that the morphing of the wing enables a significant increase in the thrust and propulsive efficiency. Du et al [19] studied the effects of unsteady deformation of a flapping model insect wing on its aerodynamic force production by solving the Navier-Stokes equations on a dynamically deforming grid. They found that with a deformation of $6 \%$ camber and $20^{\circ}$ twist, lift is increased by $10 \% \sim 20 \%$ and lift-to-drag ratio by around $10 \%$ compared with the case of a rigid flat-plate wing. Kang et al [20]studied the effects of chordwise, spanwise, and isotropic flexibility on the force generation and propulsive efficiency of flapping wings. They discovered that the maximum propulsive force is obtained when the flapping frequency is near the resonance frequency, whereas the optimal propulsive efficiency is reached when flapping frequency at about half of the natural frequency. DiPalma and Gandhi [21] proposed a new autonomous morphing helicopter rotor blade using Integrated Shape Memory Alloys. They discovered that the rotor recovered up to $43 \%$ of the lift loss at high temperature when the SMA camber morphing section extends from the blade root to $50 \%$ span. Besides, if the camber-morphing section is further extended to $75 \%$ span, up to $82 \%$ of the lost lift can be recovered. However, the passive twist considered in previous study was mainly based on elastic deformation of the flapping wings/revolving wings, hence relatively small compared with an active twist driven by a flapping mechanism at the cost of additional linkage and weight. Thus, a passive or adaptive twist angle variation for the benefit of aerodynamic performance need investigation especially for the novel FWR. Besides, in previous study, the free flight of the FWR was only achieved in the study of Dong et al [22]. They achieved the free flight of the FWR by applying 3 wings. The usage of a pitching-angle variation mechanism can greatly improve the lift efficiency of the FWR, providing an alternative way to fulfil the vertical take-off and free flight of the FWR by using 2 wings.

Thus, in this current study, an FWR driven by a coreless motor was designed, manufactured and tested to evaluate the effect of pitching angle variation on the FWR's aerodynamic forces in a flapping cycle. A special sleeve-pin unit as part of a U-shape flapping mechanism was proposed to realize the pitching angle variation of the flapping wings during up-stroke and down-stroke. The kinematics of motion of the FWR was measured using laser velocimeter and videogrammetery. A force measuring system including the load cell, signal amplifier, signal acquisition card and PC was also built to measure the total force including inertial force and aerodynamic lift of the FWR. Apart from the experimental model and measuring system, an ADAMS model of the flapping mechanism was also created to calculate the FWR's moment of inertia and inertial force, assisting the analysis of the experimental results. The effect of the passive pitching angle variation (PPAV) as well as the wing weight on the FWR's aerodynamic performance was evaluated through experiment by comparing the FWR performance at different weights, input voltage and flapping kinematics (constant pitching angle).

\section{The Flapping Wing Rotor Test Model AND NUMERICAL SIMULATION METHOD}

\section{A. Flapping wing rotor test model}

In the present study, a flapping wing rotor (FWR) model of $18.7 \mathrm{~g}$ (excluding wires) weight powered by a motor of $5.5 \mathrm{~g}$ weight was designed and manufactured with details as shown in Figure 1 and Table.1. Most components of the FWR model (in blue) as shown in Figure 1 (a) were made of highperformance nylon by $3 \mathrm{D}$ printing and the rods (in black) were made of carbon/epoxy composite. The wings' membrane was made of $12.5 \mu \mathrm{m}$ polyimide film. The rated continuous power, rated voltage and rated speed of the customized coreless motor was $15 \mathrm{~W}, 7.4 \mathrm{~V}$ and $30000 \mathrm{rpm}$ respectively. In order to provide adequate driving force, refer to the previous paper by Dong [22], a double reduction gear unit of gear ratio 22.75:1 was designed as shown in Figure 1 (b). Through the gear set and connections made of aluminium, the rotation of the coreless motor was transformed to the linear reciprocating motion of the 
"pushrod" and then transformed to the FWR's flapping motion $(\phi)$ by the U-shape mechanism. Due to the phenomenon of inverse Karmen vortex, the flapping motion would produce axial symmetric thrust and a wing rotary motion $(\psi)$.

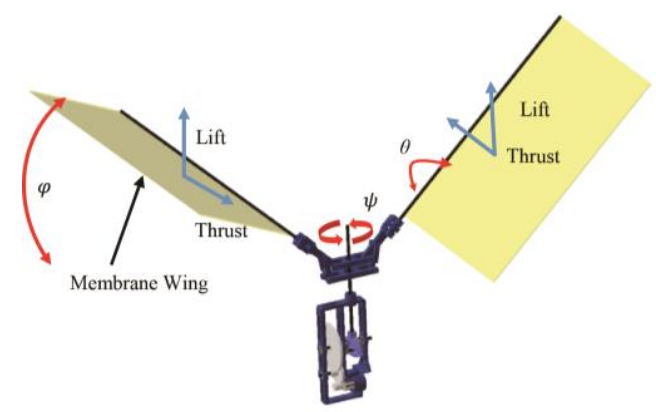

(a)

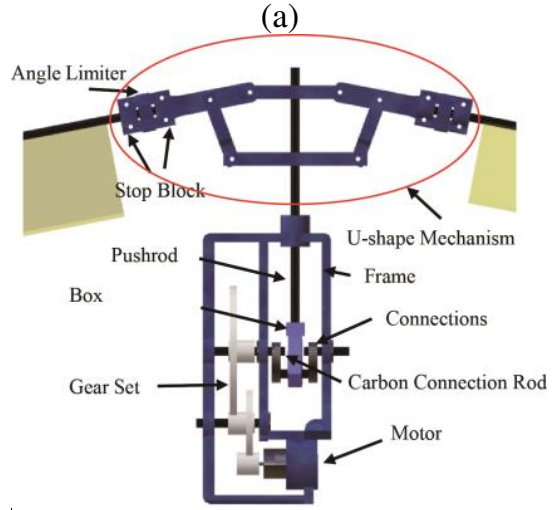

(b)

Figure 1. (a) Working principle (b) flapping mechanism of the flapping wing rotor.

The details of the U-shape mechanism of the FWR model was shown in Figure 2. On each side of wing assembly, the wing spar root was inserted like a pin into a sleeve to form a sleeve-pin unit mounted at the free end of the U-shape mechanism. This unit allows the wing free rotate about $x_{w}$ axis (spar axis) but limited by a couple of 'angle limiter' stopper as part of the sleeve. In this model, the maximum variation range of rotation angle was limited to $\pm 20^{\circ}$. During flapping motion, the wing rotates at twist angle $(\theta)$ including two parts, the rigid free pitching and elastic deformation twist in a passive manner caused by the aerodynamic pressure and inertial force acting on the wing.

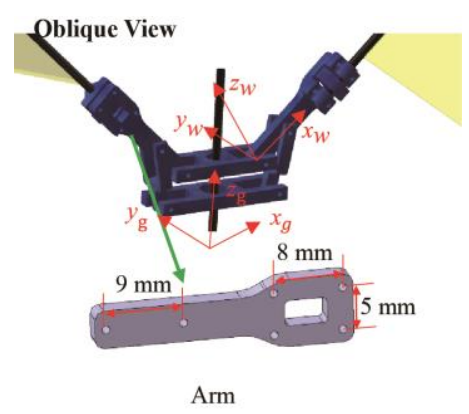

(a)



(b)

Figure 2. (a) sleeve-pin unit (b) the U-shape mechanism.

The details of the FWR model and components are listed in Table 1. Two flapping wings of the same dimension and structure layout but different weight, Wing A and Wing B, were manufactured and used in the study with further details as shown in Figure 3. The wing platform is chosen to be a rectangle since the FWR is the combination of the flapping wing and rotary wings and the rectangle wings are commonly used in most rotorcraft. A bio-inspired wing platform is not designed in this study and the optimization of the wing platform would be included in the next study.

Table 1. The components data of the flapping wing rotor

\begin{tabular}{c|c|c}
\hline Component Name & Weight $(\mathrm{g})$ & Quantity \\
\hline Frame & 2.8 & 1 \\
Box & 0.5 & 1 \\
Motor & 5.5 & 1 \\
Gear Set & 1.6 & 1 \\
Bearings & 0.25 & 5 \\
Pushrod & 0.2 & 1 \\
Connections & 0.25 & 2 \\
U-shape Mechanism & 2.95 & 1 \\
Wing A (Wing B) & $1.7(2.3)$ & 2 \\
\hline Total & $18.7(19.9)$ & 15 \\
\hline
\end{tabular}

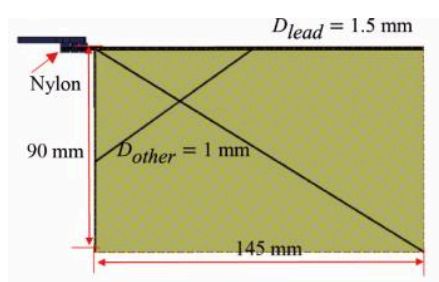

(a) Wing A-1

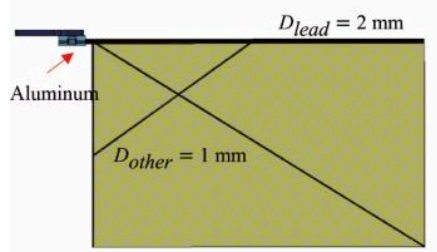

(c) Wing B-1

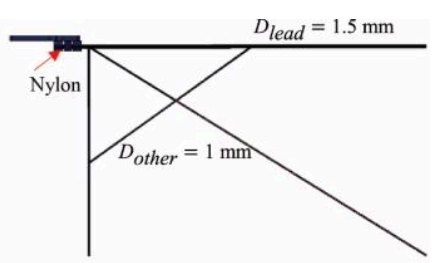

(b) Wing A-2

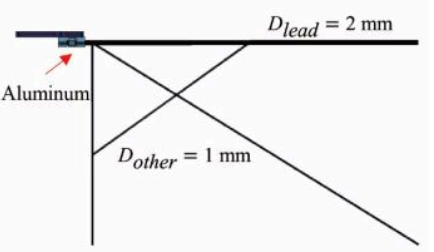

(d) Wing B-2
Figure 3. Structure layout and dimensions of the FWR model Wing A and Wing B (with and without membrane skin).

The difference between the Wing A and Wing B mainly lies in the material of the sleeve-pin unit and the diameter of the wing spar. For the Wing A assembly, the whole sleeve-pin unit was made of nylon, while for Wing B the pin arm remains as 
nylon, but the stop blocks and angle limiter were made of aluminum. The Wing A-2 and B-2 without membrane skin was for the measurement of the flapping wing inertial force.

\section{B. Setup of experimental devices}

A measurement system was built to capture the kinematics of motion and measure the corresponding lift produced by the FWR. Assuming the FWR average rotary speed is constant [23]-[25], a trace line diagram of the FWR trailing edge during a flapping motion is illustrated in Figure 4. Wherein, the green line stands for the trace line of the wing with pitching angle variation while the red line for the wing of constant pitching angle $\left(30^{\circ}\right)$. The reason for setting the pitching angle as $30^{\circ}$ is that the FWR can produce the maximum average lift at this constant angle[25]. It is feasible to measure the average rotary speed through calculating the average revolutions per second (rps) of the FWR rotary motion captured by video frames. Besides, a digital tachometer (DM6230) was used to measure the flapping frequency.

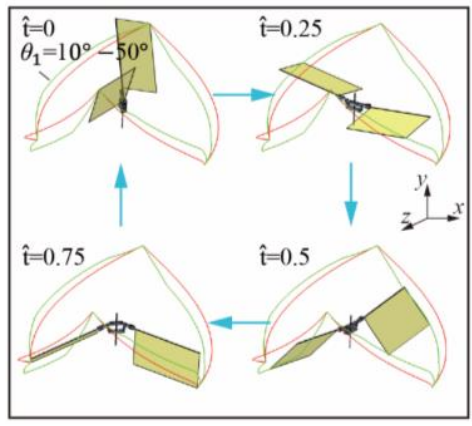

(a)

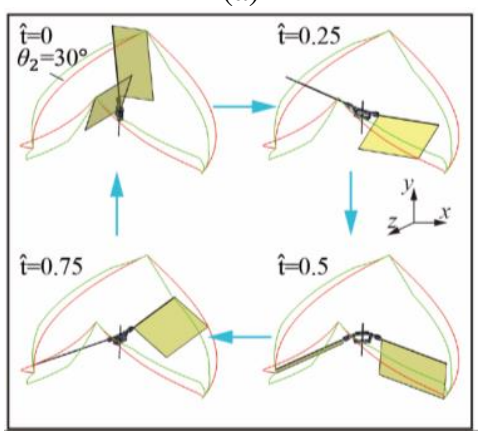

(b)

Figure 4. Trace line diagram of the FWR as well as the measurement method of flapping motion (a) pitching angle variation (b) constant pitching angle.

Figure 5 shows a typical kinematics of flapping motion of the FWR of PPAV with the maximum stroke angle $\phi=50^{\circ}$ and minimum stroke angle $\phi=-20^{\circ}$. Since the aerodynamic pressure and inertial force in $z_{g}$ direction (as shown in Figure 2) was negative in the first half of up-stroke, the pitching angle of the wing increased rapidly up to maximum limit at $\theta_{1}=50^{\circ}$ and kept the angle in the rest of up-stroke. In down-stroke, the wing rotated in opposite direction and reached the minimum pitching angle $\theta_{1}=10^{\circ}$.

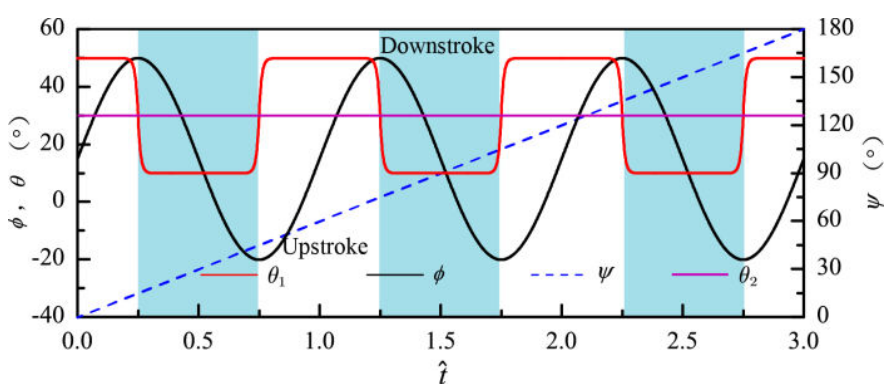

Figure 5. Kinematics of motion of the FWR of PPAV

The force measurement was operated based on a load cell (SEEED STUDIO 314990000) as shown in Figure 6. The voltage signal output from the load cell was amplified by a signal amplifier and then transferred through a signal acquisition card (NI USB-6008) and finally to a PC for data process. The test data were filtered by the 2 nd order low-pass Chebyshev algorithm in the LabVIEW code and the low-pass cutoff frequency was set as 5 times the flapping frequency of the FWR model [26], [27]. The effectiveness of the force measurement system was validated in the previous study[25].

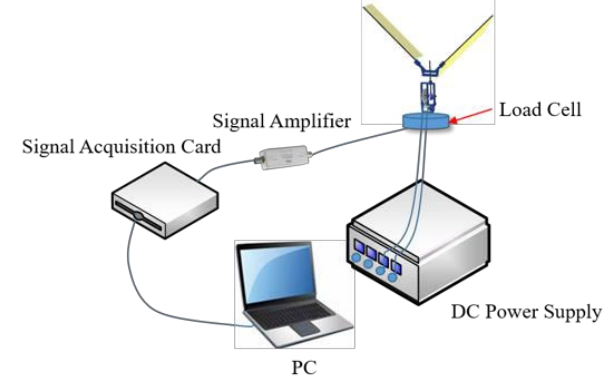

Figure 6. Force measurement system for the FWR model

Table 2. Test cases with different parameter and data setting

\begin{tabular}{cccc|cccc}
\hline Case 1 & $\theta_{\text {rigid }}$ & $V_{\text {input }}$ & $W A$ & Case 2 & $\theta_{\text {rigid }}$ & $V_{\text {input }}$ & $W A$ \\
\hline \hline $1-1$ & $30^{\circ}$ & 3 & Wing A & $2-1$ & $10^{\circ}-50^{\circ}$ & 3 & Wing A \\
$1-2$ & $30^{\circ}$ & 3.5 & Wing A & $2-2$ & $10^{\circ}-50^{\circ}$ & 3.5 & Wing A \\
$1-3$ & $30^{\circ}$ & 4 & Wing A & $2-3$ & $10^{\circ}-50^{\circ}$ & 4 & Wing A \\
$1-4$ & $30^{\circ}$ & 4.5 & Wing A & $2-4$ & $10^{\circ}-50^{\circ}$ & 4.5 & Wing A \\
$1-5$ & $30^{\circ}$ & 5 & Wing A & $2-5$ & $10^{\circ}-50^{\circ}$ & 5 & Wing A \\
$1-6$ & $30^{\circ}$ & 5.5 & Wing A & $2-6$ & $10^{\circ}-50^{\circ}$ & 5.5 & Wing A \\
$1-7$ & $30^{\circ}$ & 6 & Wing A & $2-7$ & $10^{\circ}-50^{\circ}$ & 6 & Wing A \\
$1-8$ & $30^{\circ}$ & 6.5 & Wing A & $2-8$ & $10^{\circ}-50^{\circ}$ & 6.5 & Wing A \\
\hline
\end{tabular}




\begin{tabular}{cccc|cccc}
\hline Case 3 & $\theta_{\text {rigid }}$ & $V_{\text {input }}$ & $W A$ & Case 4 & $\theta_{\text {rigid }}$ & $V_{\text {input }}$ & $W A$ \\
\hline \hline $3-1$ & $30^{\circ}$ & 3 & Wing B & $4-1$ & $10^{\circ}-50^{\circ}$ & 3 & Wing B \\
$3-2$ & $30^{\circ}$ & 3.5 & Wing B & $4-2$ & $10^{\circ}-50^{\circ}$ & 3.5 & Wing B \\
$3-3$ & $30^{\circ}$ & 4 & Wing B & $4-3$ & $10^{\circ}-50^{\circ}$ & 4 & Wing B \\
$3-4$ & $30^{\circ}$ & 4.5 & Wing B & $4-4$ & $10^{\circ}-50^{\circ}$ & 4.5 & Wing B \\
$3-5$ & $30^{\circ}$ & 5 & Wing B & $4-5$ & $10^{\circ}-50^{\circ}$ & 5 & Wing B \\
$3-6$ & $30^{\circ}$ & 5.5 & Wing B & $4-6$ & $10^{\circ}-50^{\circ}$ & 5.5 & Wing B \\
$3-7$ & $30^{\circ}$ & 6 & Wing B & $4-7$ & $10^{\circ}-50^{\circ}$ & 6 & Wing B \\
$3-8$ & $30^{\circ}$ & 6.5 & Wing B & $4-8$ & $10^{\circ}-50^{\circ}$ & 6.5 & Wing B \\
\hline
\end{tabular}

\section{Establishment of the ADAMS model}

In order to evaluate the moment of inertial about $z_{g}$ axis of the FWR in the experiment, an ADAMS model was built up as shown in Figure 7. The ADAMS model was validated by using the measured inertial force in $z_{g}$ direction of the FWR without membrane skin as shown in Figure 3 (b) and (d). Subsequently the validated ADAMS model was used to calculate the moment of inertial about $z_{g}$ for the FWR with the skin during flapping motion.

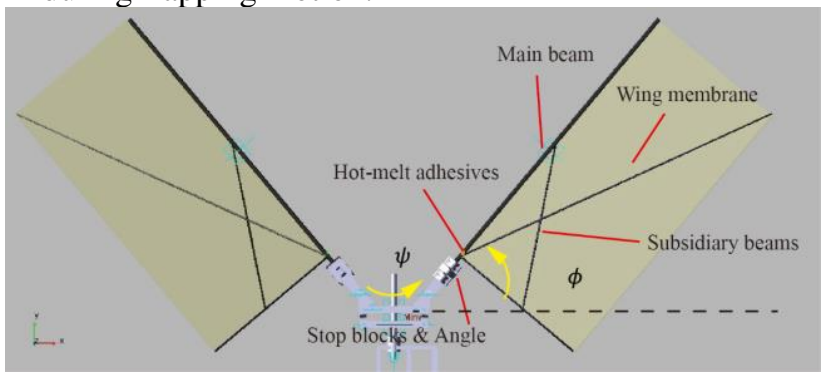

Figure 7. ADAMS model of the flapping wing rotor.

\section{EXPERIMENT RESULTS AND DISCUSSION}

\section{A. The experimental cases and data}

In order to study the effect of the PPAV and the wing weight on the FWR aerodynamic performance, number of test cases with different input voltage $\left(V_{\text {input }}\right)$, wing weight $(W A)$ and pitching angles were set in the experiment. As listed in Table 2, the maximum voltage set in the cases was $6.5 \mathrm{~V}$. It is because a higher voltage may cause the local stress concentration in the FWR's structure and wreck the carbon connection rod (as shown in Figure 1) when the FWR is mounted on the load cell (this study did not involve in the structural analysis for the skeleton). However, according to the experiment test, the FWR structure can sustain $10 \mathrm{~V}$ input voltage during its free flight test when no external force constraint was applied to the FWR. Also, the average twisting angles in Cases 1, Cases 2, Cases 3 and Cases 4 are all set to be $30^{\circ}$ since the flapping wing rotor share the highest lift efficiency under this average twisting angle when the average twisting angle is a constant value during the flapping process[14], [25]. According to our previous experiment and theoretical results[14], [25], it is because the $30^{\circ}$ average twisting angle would make the Strouhal number of the flapping wing rotor approach 0.3 that help the flapping wing rotor get the optimal lift efficiency.

\section{$B$. The total force, inertial force and lift results}

Using the measuring method depicted above, the total force including the inertial and aerodynamic lift forces in the test Case 1-7, Case 2-3, Case 3-7 and Case 4-6, which had approximately the same flapping frequency around $5.8 \mathrm{~Hz}$ were measured and shown in Figure 8. In the figure, T, In, InSim and $\mathrm{L}$ stands for the measured total force and inertial force, the calculated inertial force and the resulting lift force of the FWR model, respectively. The force-time curves started at the beginning of up-stroke when maximum negative inertial force occurred. The phase difference between the inertial force and lift force was about $90^{\circ}$ since the maximum acceleration occurred at the start of down-stroke while the aerodynamic lift reached nearly zero at this moment. According to Figure 8(a), for the Case 1-7, the instantaneous peak value of total force, inertial force and lift force is around $0.371 \mathrm{~N}, 0.141 \mathrm{~N}$ and 0.41 $\mathrm{N}$ respectively. In the Case 1-7 where the constant pitching angle was set at $30^{\circ}$, the measured average lift was only 0.0743 $\mathrm{N}$ since the negative lift was close to the positive lift although a large peak lift was found and the average inertial force over a flapping cycle was almost zero. It was noted that stroke angle was $15^{\circ}$ instead of $0^{\circ}$ in the mid-down-stroke as marked by point B in Figure 8(a). It was not until the inertial force became negative when the stroke angle reached $0^{\circ}$ at point $\mathrm{C}$. The maximum lift occurred at the point $\mathrm{D}$ between the point $\mathrm{C}$ and B.

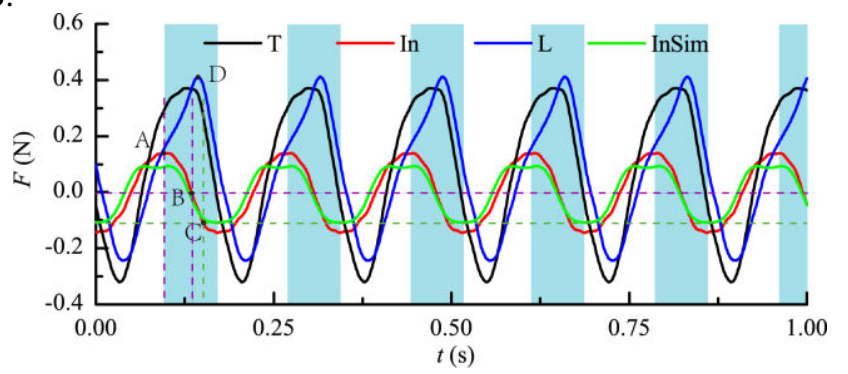

(a) 


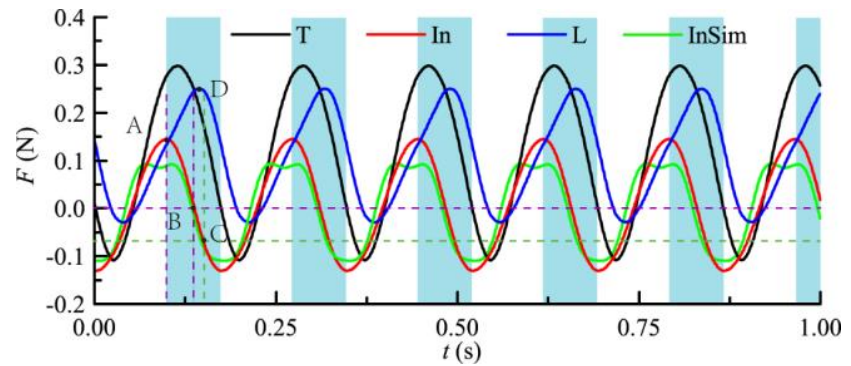

(b)

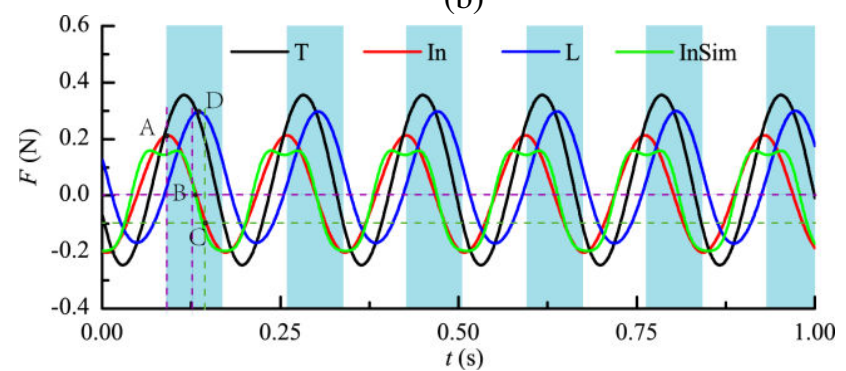

(c)

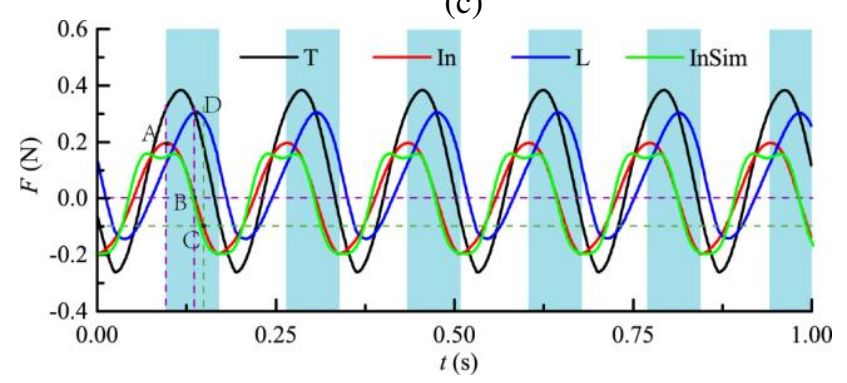

(d)

Figure 8. Total Force, Inertial force and lift force of the FWR model in (a) Case 1-7; (b) Case 2-3; (c) Case 3-7; (d) Case 4-6.

In the case $2-3$ where a PPAV was set as $10^{\circ}-50^{\circ}$, Figure

8(b) shows that the instantaneous peak value of total force, inertial force and lift force is around $0.297 \mathrm{~N}, 0.138 \mathrm{~N}$ and 0.249 $\mathrm{N}$ respectively. In this case, the negative total force was reduced significantly during the up-stroke, so as the negative lift close to $-0.03 \mathrm{~N}$. The resulting average lift of $0.103 \mathrm{~N}$ was much larger than the Case 1-7 since most of negative total force was due to the inertial force, which was cancelled out.

When the lighter Wing A $(1.7 \mathrm{~g})$ was replaced by the heavier Wing B (2.3g) in the Case 3-7, the measured maximum total force, inertial force and lift force were $0.355 \mathrm{~N}, 0.213 \mathrm{~N}$ and $0.308 \mathrm{~N}$ respectively. Meanwhile, for the Case 4-6, the measured maximum total force, inertial force and lift force were $0.382 \mathrm{~N}, 0.196 \mathrm{~N}$ and $0.305 \mathrm{~N}$ respectively. The force variation trend for the Case 4-6 was found similar to that for the Case 23 but apparent negative lift occurred in the Case 4-6. Despite the input voltage for the FWR in Case 4-6 was higher, a smaller average lift of $0.077 \mathrm{~N}$ was achieved than the lighter FWR in Case 2-3 (0.103 N).

According to Figure 8, similar peaks of the inertial forces are found in Case 1-7 and Case 2-3. Similarly, Case 3-7 and Case 4-6 shares approximately the same peaks of the inertial forces. Besides, the variation of the inertial force calculated from the ADAMS model agreed with the experimental data.
The instantaneous peak value of the inertial forces from the ADAMS $(-0.1074 \mathrm{~N})$ and experiment $(-0.144 \mathrm{~N})$ were different in Case 1-7 as shown in Figure 8(a). The inertial peak value difference was much smaller in Case 2-3 (-0.11 N and -0.132 $\mathrm{N})$, Case 3-7 (-0.195 N and -0.201 N) and Case 4-6 (-0.195 N and $-0.198 \mathrm{~N}$ ) as shown in Figure 8(b), Figure 8(c) and Figure $8(\mathrm{~d})$. The difference between the two methods was mainly due to the FWR elastic deformation in the experiment that was not considered in the ADAMS model. Through the comparison between ADAMS results and experimental data as above, the ADAMS model is validated. According to the simulation results from the ADAMS model, when the flapping angle was zero, the moment of inertia of the U-shape mechanism for the wing A and wing B models about $z_{g}$-axis was $3.67 \mathrm{e}-5 \mathrm{~kg} \cdot \mathrm{m}^{2} / \mathrm{s}$ and $5.28 \mathrm{e}-5 \mathrm{~kg} \cdot \mathrm{m}^{2} / \mathrm{s}$ respectively.

\section{The effect of passive pitching angle on the FWR motion and efficiency}

The measurement results of the FWR motion and forces against the input power for the three cases listed in Table 2 are plotted in Figure 9. From Figure 9(a) and Figure 9(b), it can be found that similar flapping frequencies and rotary speeds for the Cases 1, 2, 3 and 4 were obtained under the same input voltage. Compared with Cases 1, Cases 3 and Cases 4, significantly higher flapping frequency and rotary speed are found in Cases 2. In particular, the rotary speed $(6.75 \mathrm{rev} / \mathrm{s})$ in Case 2-8 is more than 5 times higher than the Case 3-8 (1.25 rev/s).

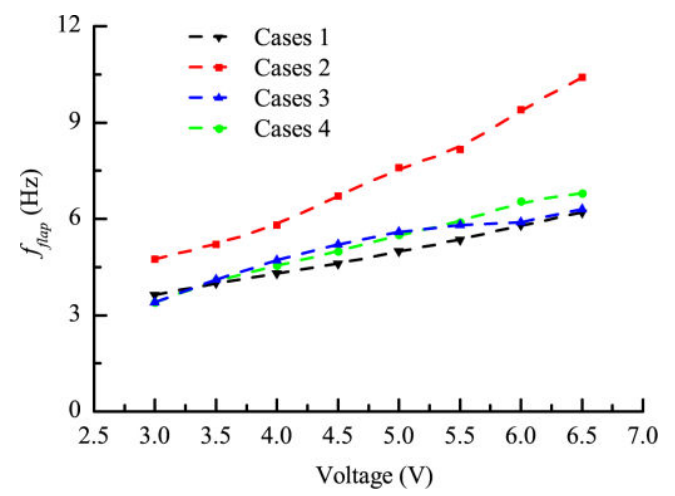

(a)

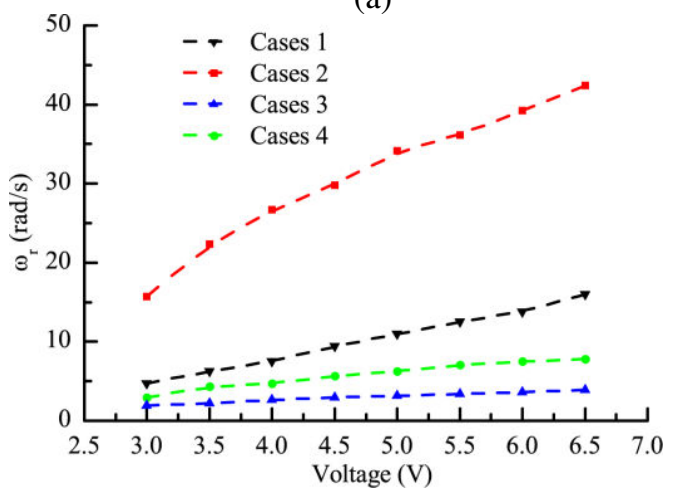

(b) 


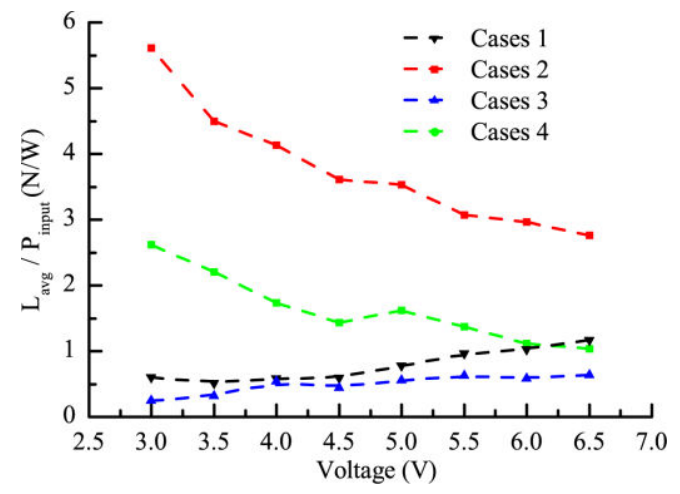

(c)

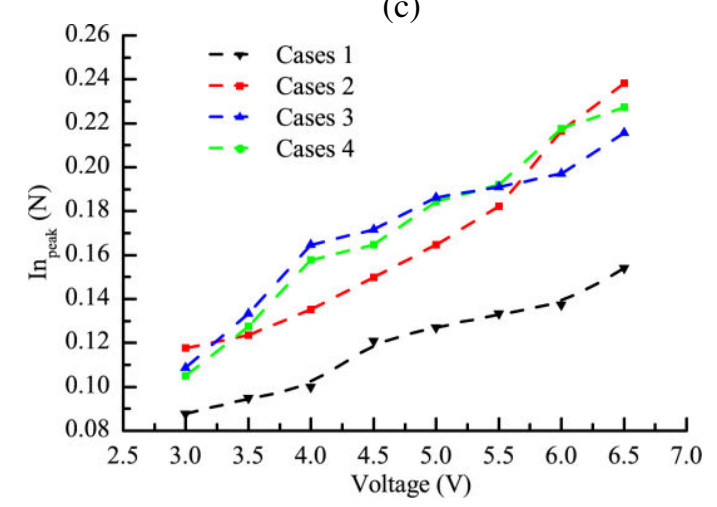

(d)

Figure 9. (a) Flapping frequency; (b) Rotary speed; (c) Lift-topower ratio; (d) Peak values of the inertial force of the FWR in a range of input voltage.

As shown in Figure 9(c), the lowest lift-to-power ratio was found for the Cases 3 where the constant pitching angle was set and Wing B is used. On the other hand, under the same input voltage, the FWR of PPAV in Cases 2 share the highest aerodynamic efficiency (over twice than that in Cases 1, Cases 3 and Cases 4). According to the previous studies [14], [25], optimal average twisting angle of the FWR should be $30^{\circ}$ (as given in both Cases 2 and Cases 4) and thus the poor performance of Cases 4 is mainly due to its larger weight. The results demonstrated that a pair of lighter wings (Wing A) would have higher efficiency. Besides, it is found that despite the lift-to-power ratio increases with the increase of the input voltage in Cases 1 and Cases 3, the lift-to-power ratio in Cases 2 and Cases 4 keep decreasing with the increase of the input voltage. As depicted in previous subsection, the maximum voltage set in the cases was $6.5 \mathrm{~V}$ because a higher voltage may cause the local stress concentration in the FWR's structure and wreck the carbon connection rod (as shown in Figure 1) when the FWR is mounted on the load cell. Due to this fact, we did not contribute the optimization work in this study to find the optimized input voltage.

As shown in Figure 9 (d), although the peak values of the inertial forces in Cases 2 is significantly higher than those in Cases 1 in the range of the input voltage, the FWR shares higher peaks of inertial forces in the Cases 1 under the same flapping frequency. The peak inertial forces in the Cases 2, Cases 3 and Cases 4 show a small difference as shown in Figure 9 (d) under the same input voltage. Also, it can be found that the peak inertial force in Cases 4 are about 1.35 times of the Cases 2 at the same flapping frequency. This figure approximately equals to the weight ratio of the wing B to wing A $(2.3 \mathrm{~g} / 1.7 \mathrm{~g})$.

Through the above analysis, the heavier wing would require more power to overcome the inertial force and provide less power to gain higher flapping frequency. Besides, the heavier wing B had larger moment of inertia about $z_{g}$ axis (as given in the previous subsection) thus led to lower rotary speed.

\section{$D$. The effect of passive pitching angle on the FWR average lift}

The measured average aerodynamic lift $\left(\mathrm{L}_{\text {avg }}\right)$ together with the average total force $\left(\mathrm{T}_{\text {avg }}\right)$ and average inertial force $\left(\mathrm{In}_{\text {avg }}\right)$ results for the Cases 1, Cases 2, Cases 3 and Cases 4 are plotted in Figure 10. As the input voltage increased, the total force and lift increased rapidly but the average inertial force kept nearly zero. Among all the cases, the smallest average lift was found in Cases 3. Under the same input voltage, the average lift of the FWR in Cases 2 is more than 3 times of that in Cases 3. Besides, under any of the input voltage, the average lift in the Cases 2 are twice of those in the Cases 4. Also, the lift-voltage relationship for the FWR of PPAV is almost linear.

Generally, the average lifts produced by the FWR model of PPAV in the Cases 4 are significantly higher than the opponent of constant pitching angle $\left(30^{\circ}\right)$ in the Case 3 while the lifts in Case 4 are still not enough to overcome the weight of the flapping wing rotor. The less weight of the wing would generate smaller inertial forces at the same flapping frequency since $F_{\text {inertial }}=-m_{\text {wing }} a_{\text {vertial }}$ and thus we tried to make a pair of lighter wings (such as Wing A) to help generate higher lift efficiency (as shown in Figure 9 (c)).



(a)

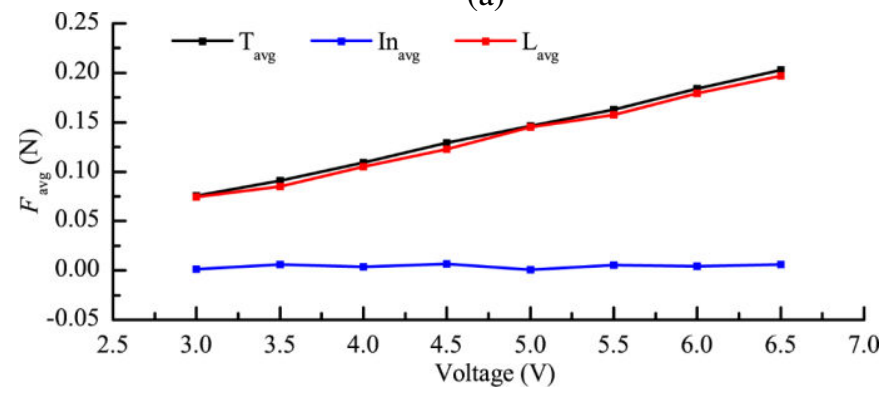

(b) 


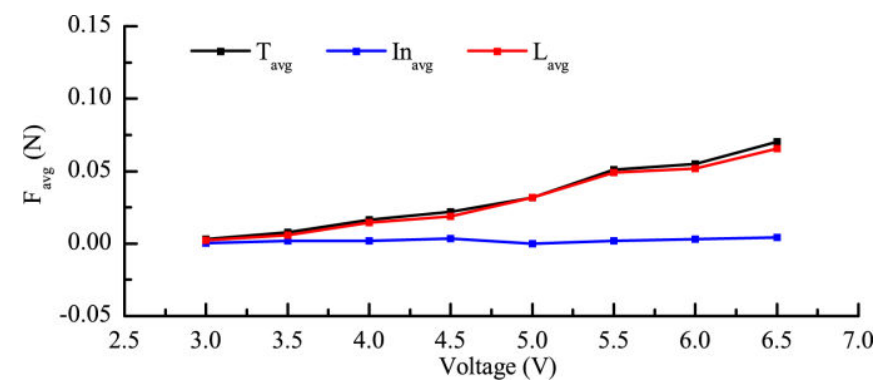

(c)

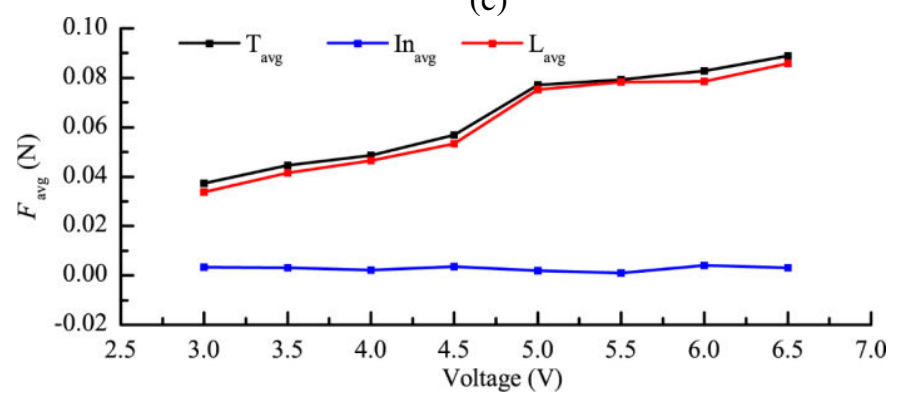

(d)

Figure 10. Measurement of average force for the FWR. (a) Average force for Cases 1; (b) Average force for Cases 2; (c) Average force for Cases 3; (d) Average force for Case 4.

From the data in Table 1, it is found that the net weight of the FWR model (excluding the wire) is $18.7 \mathrm{~g}$ with wing A or $20.1 \mathrm{~g}$ with wing B. According to Figure 10, the maximum average lift $(0.201 \mathrm{~N})$ with a lift-to-weight ratio 1.07 was achieved for the Case 2-8. The result indicates that it is feasible for the FWR model to realize a vertical take-off flight.

\section{E. A vertical take-off free flight of the flapping wing rotor}

In order to prove the FWR model's feasibility of vertical take-off and free flight, a wire of about $3 \mathrm{~m}$ long and $4 \mathrm{~g}$ weight was used to connect an external power supply to the model.



Figure 11. Snapshots of the free-flight test of the FWR model.

It was found that the minimum required input voltage to provide enough power to lift the $22.7 \mathrm{~g}$ weight ( $18.7 \mathrm{~g}$ FWR plus $4 \mathrm{~g}$ cable) was $9 \mathrm{~V}$ as shown in Figure 11. The distance from the FWR model at 0 s to the ceiling of the room is $1.3 \mathrm{~m}$. Among the snapshots during the FWR free flight over $2 \mathrm{~s}$, it was found that after releasing the FWR at $0.167 \mathrm{~s}$, the FWR reached the room ceiling at about $1 \mathrm{~s}$ flight. Since no flight control was applied, the FWR model behaved unstable and eventually fallen down to the ground. Nevertheless, the test shows that the Ushape mechanism with a novel sleeve-pin unit used in the FWR model can achieve PPAV and lead to significant increase of aerodynamic lift and performance than a FWR of constant pitching angle.

\section{CONCLUSIONS}

In the present investigation, a novel sleeve-pin unit mounted the U-shape flapping mechanism was proposed for an FWR to achieve large pitching angle variation (PPAV) during flapping motion. The significance of the PPAV for improving the FWR aerodynamic performance has been proven through design, manufacture, experiment and analysis of an FWR test model. In order to evaluate the PPAV effect, the FWR models of different wings and kinematics of motion were manufactured and tested. The model with a pair of flapping wings of constant pitching angle $30^{\circ}$ setting was taken as the baseline for comparison according to the previous study[14], [25]. From the study, the following remarks are drawn.

- The proposed sleeve-pin unit is simple and yet very effective to achieve a required kinematics of flapping motion for the FWR;

- The pre-defined FWR maximum $\left(50^{\circ}\right)$ and minimum angle $\left(10^{\circ}\right)$ can be achieved in a passive manner by making use of the aerodynamic pressure and inertia force during up-stroke and down-stroke.

- The negative aerodynamic lift produced by the FWR of PPAV during up-stroke is reduced significantly in up-stroke comparing with the FWR of a constant pitching angle. This resulted in a large increase of aerodynamic average lift and efficiency.

- A lighter wing will not only reduce the inertia force and power demand, but also produce higher flapping frequency and lift under the same input power comparing with a heavier wing;

- The inertial force of the FWR can be calculated by using a numerical model based on the design of the test model and validated by using the test data. The net aerodynamic force can be extracted from the measured total force minus the inertia force.

- A free flight of the FWR model has been demonstrated to prove the PPAV effectiveness for the FWR.

\section{REFERENCES}

[1] L. J. Roberts, H. A. Bruck, and S. K. Gupta, "Modeling of dive maneuvers in flapping wing unmanned aerial vehicles," SSRR 2015 - 2015 IEEE Int. Symp. Safety, Secur. Rescue Robot., 2016. 
[2] J. S. Izraelevitz, Q. Zhu, and M. S. Triantafyllou, "StateSpace Adaptation of Unsteady Lifting Line Theory: Twisting/Flapping Wings of Finite Span," AIAA J., vol. 55, no. 4, pp. 1279-1294, 2017.

[3] R. Madangopal, Z. A. Khan, and S. K. Agrawal, "Energetics-based design of small flapping-wing micro air vehicles," IEEE/ASME Trans. Mechatronics, vol. 11, no. 4, pp. 433-438, 2006.

[4] S. Mishra, B. Tripathi, S. Garg, A. Kumar, and P. Kumar, "Design and Development of a Bio-Inspired Flapping Wing Type Micro Air Vehicle," in International Conference on Nanomaterial's and Technologies, 2015, pp. 519-526.

[5] L. Hines, V. Arabagi, and M. Sitti, "Shape Memory Polymer-Based Flexure Stiffness Control in a Miniature Flapping-Wing Robot," IEEE Trans. Robot., vol. 28, no. 4, pp. 987-990, 2012.

[6] S. S. Baek, Fernando L.G.B, and R. S. Fearing, "Flight control for target seeking by 13 gram ornithopter," in IEEE International Conference on Intelligent Robots and Systems, 2011, pp. 2674-2681.

[7] G. K. Lau, Y. W. Chin, J. T. W. Goh, and R. J. Wood, "Dipteran-insect-inspired thoracic mechanism with nonlinear stiffness to save inertial power of flapping-wing flight," IEEE Trans. Robot., vol. 30, no. 5, pp. 1187-1197, 2014.

[8] A. K. Stowers and D. Lentink, "Folding in and out: passive morphing in flapping wings," Bioinspir. Biomim., vol. 10, no. 2, p. $025001,2015$.

[9] L. Zheng, T. L. Hedrick, and R. Mittal, "Time-Varying Wing-Twist Improves Aerodynamic Efficiency of Forward Flight in Butterflies," PLoS One, vol. 8, no. 1, pp. 1-10, 2013.

[10] V. Arabagi, L. Hines, and M. Sitti, “A Simulation and Design Tool for a Passive Rotation Flapping Wing Mechanism," IEEE/ASME Trans. MECHATRONICS, vol. 18, no. 2, pp. 787-798, 2013.

[11] Z. Yan, H. E. Taha, and M. R. Hajj, "Effects of aerodynamic modeling on the optimal wing kinematics for hovering MAVs," Aerosp. Sci. Technol., vol. 45, pp. 39-49, 2015.

[12] D. Li, S. Guo, N. D. Matteo, and D. Yang, "Design, Experiment and Aerodynamic Calculation of a Flapping Wing Rotor Micro Aerial Vehicle," in 52nd AIAA/ASME/ASCE/AHS/ASC Structures, Structural Dynamics and Materials Conference, 2011, pp. 1-9.

[13] C. Zhou, J. Wu, S. Guo, and D. Li, "Experimental study on the lift generated by a flapping rotary wing applied in a micro air vehicle," Proc IMechE Part G J. Aerosp. Eng., vol. 228, no. 11, pp. 2083-2093, 2014.

[14] H. Li and S. Guo, "Aerodynamic efficiency of a bioinspired flapping wing rotor at low Reynolds number," R. Soc. Open Sci., vol. 5, no. 3, pp. 1-15, 2018.

[15] Z. W. Guan and Y. L. Yu, "Aerodynamic mechanism of forces generated by twisting model-wing in bat flapping flight," Appl. Math. Mech. (English Ed., vol. 35, no. 12, pp. 1607-1618, 2014.

[16] T. Q. Truong, V. H. Phan, H. C. Park, and J. H. Ko, "Effect of Wing Twisting on Aerodynamic Performance of Flapping Wing System," AIAA J., vol. 51, no. 7, pp. 1612-1620, 2013.

[17] F. Sal, "Effects of the actively morphing root chord and taper on helicopter energy," Aircr. Eng. Aerosp. Technol., vol. 92, no. 2, pp. 264-270, 2020.

[18] M. Ghommem, M. R. Hajj, P. S. Beran, and I. K. Puri, "Role of wing morphing in thrust generation," Theor. Appl. Mech., vol. 4, no. 3, pp. 1-7, 2014.
[19] G. Du and M. Sun, "Effects of unsteady deformation of flapping wing on its aerodynamic forces," Appl. Math. Mech. (English Ed., vol. 29, no. 6, pp. 731-743, 2008.

[20] C.-K. Kang, H. Aono, C. E. S. Cesnik, and W. Shyy, "Effects of flexibility on the aerodynamic performance of flapping wings," J. Fluid Mech, vol. 689, no. June, pp. 3274, 2011.

[21] M. DiPalma and F. Gandhi, "Autonomous camber morphing of a helicopter rotor blade with temperature change using integrated shape memory alloys," J. Intell. Mater. Syst. Struct., vol. 32, no. 5, pp. 499-515, 2021.

[22] X. DONG, D. LI, J. XIANG, and Z. WANG, "Design and experimental study of a new flapping wing rotor micro aerial vehicle," Chinese J. Aeronaut., vol. 33, no. 12, pp. 30923099, 2020.

[23] J. Wu, D. Wang, and Y. Zhang, "Aerodynamic Analysis of a Flapping Rotary Wing at a Low Reynolds Number," AIAA J., vol. 53, no. 10, pp. 2951-2966, 2015.

[24] D. Wang, Y. Zhang, J. Wu, and Y. Zhang, "Aerodynamics on Flapping Rotary Wing in Low Reynolds Number," in American Institute of Aeronautics and Astronautics, 2013, no. 37, pp. 1-14.

[25] S. Chen, L. Wang, S. Guo, C. Zhao, and M. Tong, “A bioinspired flappingwing rotor of variant frequency driven by ultrasonic motor," Appl. Sci., vol. 10, no. 1, pp. 1-21, 2020.

[26] K. O. Granlund, M. V. O1, and L. P. Bernal, "Unsteady pitching flat plates," J. Fluid Mech., vol. 733, no. R5, 2013.

[27] K. Ramesh, A. Gopalarathnam, J. R. Edwards, M. V. Ol, and K. Granlund, "An unsteady airfoil theory applied to pitching motions validated against experiment and computation," Theor. Comput. Fluid Dyn., vol. 27, no. 6, pp. 843-864, 2013.



Si Chen was born in 1993 in Zhenjiang, China. He received the B.Eng. and Ph.D. degrees from Nanjing University of Aeronautics and Astronautics (NUAA), Nanjing, China, in 2015 and 2020, respectively.

From 2016 to 2018, he went to the England and studied in the Cranfield University as a joint training doctoral. After graduating from NUAA, he worked as the lecturer in Wenzhou University. His research interests include flapping wing rotor, flapping wing, unsteady aerodynamic calculation and he has authored or coauthored 5 internationally refereed papers in these research interests.

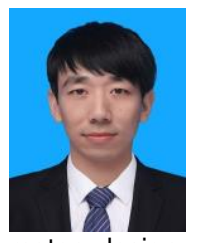

Le Wang was born in Shanxi, China. He received his Ph.D. degrees in the College of Aerospace Engineering form Nanjing University of Aeronautics and Astronautics (NUAA), Nanjing, China, in 2020.

$\mathrm{He}$ is currently a lecturer in Wenzhou University and participates two National Natural Science Foundation projects. His main research areas are novel ultrasonic motor design and bio-inspired flapping wing rotor design. He has published over 10 papers and patents in novel ultrasonic motor design and flapping wing rotor design.

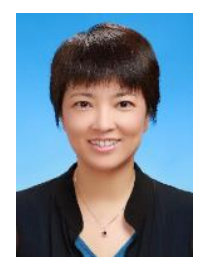

Yuanyuan He completed her Bachelor's, Master's and $\mathrm{PhD}$ degrees in Aircraft Design at Beijing Institute of Technology (BIT) in 1995, 2002, 2013, respectively. Funded by China Scholarship Council, she has visited Cranfield University as an academic visitor in 20092010. In 2015, she took a nine-months visiting at Cranfield University for a collaboration research project.

She is currently an associate professor in the School of Aerospace Engineering at BIT specialized in aircraft structures and optimization. She has two projects funded by National Natural Science Foundation of China as PI with an ongoing project on bioinspired flapping wing MAV. She has also published numbers of papers and patents in novel MAV. 


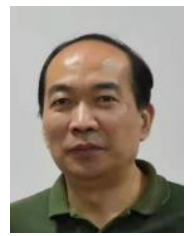

Mingbo Tong obtained his M.S. degree in solid mechanics from Northwest University of technology in 1988 and a Ph.D degree in solid mechanics from Beihang University of Aeronautics and Astronautics (BUAA) in 1995

$\mathrm{He}$ is a professor in NUAA and used to be the vice president of College of Aeronautics and Astronautics in NUAA. He is specialized in structural strength analysis, solid mechanics and aircraft design and authored or coauthored over 90 technical papers in his research area.

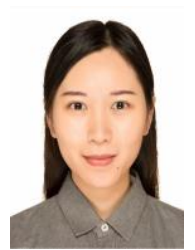

Yingjun Pan received her B.S degree in aircraft design and engineering from Harbin Engineering University, Harbin, China, in 2017, and her MSc degree in aerospace vehicle design from Cranfield University, United Kingdom, in 2019.

She worked in Aviation industry as an aerodynamic engineer in 2019 and then a Research Institute within Guangdong Academy of Sciences, China, engaged in developing a 1-seat eVTOL aircraft in 2020. She is currently a Ph.D student to study bio-inspired flapping wings at Cranfield University.

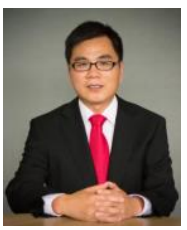

Bing Ji received the Bachelor's and Master's degrees in electronic engineering from Xidian University, China, in 2007 and 2009, respectively. He received the Ph.D. degree in medical engineering from the University of Hull, U.K., in 2012.

Since 2012, he has been a Lecturer with Shandong University, China and currently holds the associate professor position. His research interests include bionic robotics, machine learning, and computational modeling of biological systems. He supervises the National Key Research and Development Program of China and has published over 10 internationally refereed papers.

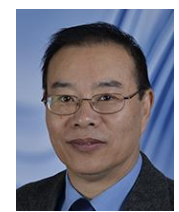

Shijun Guo received his Ph.D. degrees in aerospace engineering from University of Hertfordshire, UK, and subsequently worked in University of London and Oxford university as research fellow. He joined Cranfield University in 2003 and currently holds professor position. Prior to his academic positions, he worked in aerospace industry for over 10 years including formal British Aerospace (Civil Aircraft Division, Hatfield, 1987-92) as a trainee engineer. $\mathrm{He}$ is specialized in Aeroelasticity and Composite Structures, especially proposed the flapping wing rotor concept and initiated the research field since 2008. He has published over 140 journal and conference papers in the above research area. 
2021-09-22

\section{Aerodynamic performance of a flyable flapping wing rotor with passive pitching angle variation}

Chen, Si

IEEE

Chen S, Wang L, He Y, et al., (2022) Aerodynamic performance of a flyable flapping wing rotor with passive pitching angle variation. IEEE Transactions on Industrial Electronics, Volume 69, Number 9, September 2022, pp. 9176-9184

https://doi.org/10.1109/TIE.2021.3112964

Downloaded from Cranfield Library Services E-Repository 